\title{
¿Es diferente el seguimiento de pacientes con diabetes mellitus tipo 2 en medio rural y urbano?
}

\author{
Belén Roldán Castillo ${ }^{a}$, Candelaria Ayuso Raya ${ }^{\mathrm{b}}$, Carmen González Villora ${ }^{\mathrm{c}}$, \\ Samuel Matos Berroa ${ }^{d}$, Francisco Escobar Rabadán ${ }^{b}$
}

\begin{abstract}
a Médico de Familia. Centro de Salud Zona II de Albacete.

b Médico de Familia. Centro de Salud Universitario Zona IV de Albacete.

${ }^{c}$ Médico de Familia. Centro de Salud Zona VIII de Albacete.

d Residente de Medicina Familiar y Comunitaria. Unidad Docente de Albacete.
\end{abstract}

Correspondencia: Belén Roldán Castillo, Centro de Salud Zona II, c/ Avda de Ramón y Cajal n ${ }^{\circ}$ 24, 02005 Albacete, España, Telf: 967241616

e-mail: brolcas@gmail.com

Recibido el 14 de febrero de 2012.

Aceptado para su publicación el 10 de marzo de 2012.

\section{RESUMEN}

Objetivo. Conocer la variabilidad en el cumplimiento de los protocolos de seguimiento de pacientes diagnosticados de diabetes mellitus tipo 2 en centros de salud urbano y rural.

Diseño. Observacional, descriptivo, transversal.

Emplazamiento. Tres centros de salud de la provincia de Albacete (urbano, rural y semiurbano). Participantes. Un total de 173 pacientes diagnosticados de diabetes mellitus tipo 2 que siguieron controles rutinarios en los centros de salud seleccionados durante el año 2008. Pertenecían al centro de salud urbano 83 sujetos y 45 a cada uno de los otros dos centros. Mediciones principales. Sexo, edad, años de evolución, índice de masa corporal, fondo de ojo, electrocardiograma, microalbuminuria, hemoglobina glicosilada, perfil lipídico y hemoglobina glicosilada en rango.

Resultados. La media de edad de los pacientes al diagnóstico fue de 68,6 años (DE: 11,9) con un rango de 40 a 89 años, siendo mujeres el $45,1 \%$. No existieron diferencias en la distribución por edad y sexo en los centros de salud. En el centro de salud rural se había realizado fondo de ojo al $46,7 \%$ de los pacientes, electrocardiograma al $73,3 \%$, determinación de microalbuminuria al $37,8 \%$ y de hemoglobina glicosilada cada 6 meses al $86,7 \%$, siendo estas proporciones significativamente superiores a las de los otros centros. El $82 \%$ de los pacientes del centro rural y el $78,6 \%$ de los del semiurbano disponían de hemoglobina glicosilada $<7$.

Mediante regresión logística se identificó como única variable independiente asociada al seguimiento del protocolo el pertenecer al centro de salud rural: urbano en relación al rural OR: 0,18 (IC95\%: 0,06-0,56) y semiurbano en relación al rural OR: 0,18 (IC95\%: 0,05-0,58).

Conclusión. En el centro de salud rural existe un mayor seguimiento de los controles protocolizados que en el centro de salud urbano y semiurbano.

Palabras Clave. Diabetes Mellitus. Atención Primaria de Salud. Adhesión a directriz.

\section{ABSTRACT}

Is monitoring type II diabetes mellitus different in urban and rural environments?

Objective. To determine variability in the compliance of protocols for monitoring patients diagnosed with type II diabetes mellitus in urban and rural health centres.

Design. Observational, descriptive, cross sectional.

Location. Three health centres in the province of Albacete (urban, rural and semi-urban).

Participants. A total of 173 patients diagnosed with type II diabetes mellitus who underwent routine controls in selected health centres during the year 2008. 83 belonged to the urban centre and 45 to each of the other two centres.

Main Measures. Gender, years of progress, body mass index, inner eye, electrocardiogram, microalbuminuria, glycated haemoglobin, lipid profile and glycated haemoglobin within range.

Results. The average age of the diagnosed patients was 68,6 years (DE: 11,9), ranging from 40 to 89 years, of which $45,1 \%$ were female. There were no differences in age or gender distribution in the health centres. In the rural health centre, an inner eye test had been carried out on $46,7 \%$ of the patients, an electrocardiogram on $73,3 \%$, tests for microalbuminuria on $37,8 \%$ and for glycated haemoglobin every 6 months on $86,7 \%$, with these proportions being significantly higher than those of other centres. Glycated haemoglobin $<7$ was available to $82 \%$ of the rural centre patients and $78,6 \%$ of the semi-urban centre. Through logistic regression, the fact of belonging to the rural health centre was identified as a sole independent variable associated with protocol monitoring: urban in relation to rural OR: 0,18 (IC95\%: 0,06-0,56) and semi-urban in relation to rural OR: 0,18 (IC95\%: 0,05-0,58).

Conclusion. In the rural health centre, there is a greater monitoring of recorded controls than in the urban and semi-urban centres.

Key Words. Diabetes Mellitus. Primary Health Care. Guideline Adherence.

Una versión preliminar de este trabajo fue presentada como comunicación en formato póster en el $16^{\text {th }}$ Wonca Europe Conference, celebrado en Málaga durante los días 6-9 de Octubre de 2010. 


\section{INTRODUCCIÓN}

El término diabetes mellitus (DM) define alteraciones metabólicas de múltiples etiologías caracterizadas por hiperglucemia crónica y trastornos en el metabolismo de los hidratos de carbono, las grasas y las proteínas, resultado de defectos en la secreción de insulina, en la acción de la misma o en ambas ${ }^{1}$. La forma más frecuente es la diabetes mellitus tipo 2 (DM2), que representa el 90-95\% de los casos². La mayoría de los diabéticos tipo 2 tiene sobrepeso u obesidad, lo que contribuye a presentar un aumento en la resistencia a la insulina. Es un tipo de diabetes que presenta grados variables de déficit insulínico y resistencia periférica a la acción de la insulina. En DM 2, con frecuencia se producen elevados niveles de insulinemia inicial compensatoria, provocando a la larga una secreción insulínica insuficiente para compensar la resistencia a insulina.

Los cambios en el estilo de vida convierten a la diabetes mellitus no sólo en un problema sanitario mundial de primera magnitud, sino en una auténtica "epidemia del siglo XXI". Los nuevos hábitos de vida, fundamentalmente el exceso de ingesta calórica, la obesidad y el sedentarismo, permiten predecir un gran incremento en las próximas décadas, en especial en los países que en la actualidad están en vías de desarrollo4. Las estimaciones indican que para el año 2030 su prevalencia alcanzará proporciones epidémicas y afectará a 366 millones de personas en todo el mundo ${ }^{5}$.

La morbilidad, en términos de deterioro grave de las funciones visual y renal y de accidentes cardiovasculares, supone un porcentaje significativo de la originada por el conjunto de las enfermedades crónicas ${ }^{6}$.

La prevalencia actual de DM2 en España se sitúa en un $10-15 \%{ }^{5}$ y aumenta con la edad, hasta alcanzar el $25 \%$ en pacientes mayores de 70 años ${ }^{7}$. Más preocupante aún es el hecho de que existe alta proporción de diabéticos sin diagnosticar. En España la diabetes mellitus representa la tercera causa de muerte en las mujeres y la séptima en los varones. Es una de las pocas enfermedades que muestra una mayor mortalidad en mujeres que en varones $^{1,7-9}$.

El sistema sanitario debe conseguir que la atención al paciente con diabetes sea accesible, integral, de buena calidad, eficaz y a un coste razonable. Con- seguir este objetivo precisa del aprovechamiento de todos los recursos sanitarios disponibles, su racionalización y una coordinación efectiva y eficiente entre los distintos niveles sanitarios ${ }^{10}$.

La Atención Primaria ocupa un lugar estratégico, dada su accesibilidad y su seguimiento en el tiempo, para dar respuesta a este reto de control de enfermedades crónicas ${ }^{11}$. Con independencia de las características concretas de la organización de cada sistema sanitario, parece obvio que es en el ámbito de la atención primaria y de la comunidad donde han de desarrollarse, de forma prioritaria, tanto las actuaciones de prevención como las de seguimiento y control de los pacientes diagnosticados de diabetes mellitus tipo 2. Esta afirmación no contradice la necesidad de que exista, en forma de consultorías puntuales, una colaboración estrecha entre los profesionales médicos y de enfermería de atención familiar y comunitaria y los especialistas en endocrinología y nutrición ${ }^{6}$. Revisando la bibliografía publicada en los últimos años en relación con este tema, la mayoría de trabajos encontrados hacen referencia a la evaluación de la calidad asistencial prestada a los pacientes diabéticos tipo 2 en diferentes Áreas de Salud del territorio nacional. En algunos de ellos se compara las diferencias en el seguimiento de dichos pacientes, pero entre niveles asistenciales, como son Atención Primaria y Endocrinología ${ }^{12,13}$.

En el presente estudio pretendemos analizar si existe un adecuado seguimiento de los pacientes para evitar el desarrollo de complicaciones según las recomendaciones de las guías de práctica clínica europeas para el abordaje de la detección precoz, cribado, tratamiento y seguimiento de las complicaciones crónicas.

\section{MATERIAL Y MÉTODOS}

El estudio fue realizado en tres centros de salud de la provincia de Albacete: uno urbano (Zona IV), otro semiurbano (Tobarra) y el tercero rural (Consultorio de Villavaliente).

Se trata de un estudio observacional, descriptivo y transversal, en el que se incluyeron 173 pacientes, 83 del centro de salud urbano y 45 de cada uno de los otros, diagnosticados de diabetes mellitus tipo 2. Se siguieron controles rutinarios en los centros de salud seleccionados desde enero del 2008 a marzo del 2009. Los sujetos fueron selecciona- 
dos mediante muestreo sistemático del listado de pacientes diagnosticados de DM tipo 2 en dichos centros de salud.

Posteriormente procedimos a la revisión de las historias clínicas y recopilación de los resultados analíticos a través de los programas informáticos de Atención Primaria y del laboratorio del Complejo Hospitalario Universitario de Albacete.

Las variables estudiadas fueron:

- Cuantitativas: edad, años de evolución, índice de masa corporal (IMC), microalbuminuria, perfil lipídico y hemoglobina glicosilada .

- Cualitativas (dicotómicas): sexo y hemoglobina glicosilada en rango, fondo de ojo y electrocardiograma.

Se recogió la constancia o no de estos datos en la historia clínica.

Respecto al análisis estadístico, la medida de tendencia central utilizada fue la media y como medida de dispersión se usó la desviación estándar. Se realizó un análisis bivariante por medio del test de chi cuadrado para el contraste de variables cualitativas y el análisis de varianza para las cuantitativas, recurriendo a test no paramétricos si éstas no seguían una distribución normal. Por último, se llevó a cabo un análisis de regresión logística en el que la variable dependiente considerada fue la existencia o no de un seguimiento adecuado del protocolo. Los datos fueron analizados mediante el programa comercial SPSS 12.0.

\section{RESULTADOS}

Se estudiaron un total de 173 pacientes $(47,9 \%$ pertenecían al centro de salud urbano y $26 \%$ a cada uno de los otros dos). La media de edad de los pacientes al diagnóstico fue de 68,6 años (DE: 11,9 ), con una rango de edad 40-89 años, no observándose diferencias estadísticamente significativas entre los tres centros de salud respecto a la edad y el sexo. La distribución de las variables sociodemográficas se muestra en la tabla 1.

Se observó un control metabólico significativamente peor, valorado mediante las cifras de hemoglogina glicosilada, en el centro de salud urbano que en los centros semiurbano y rural, con medianas de 7\% (Rango intercuartílico: 6,3-8,2), 5,9\% (Rango intercuartílico: 5,2-6,5) y 6,6\% (Rango intercuartílico: $6,0-7,0)$, respectivamente $(p<0,0001)$.

Respecto al tiempo de evolución (años transcurridos desde el diagnóstico), fue significativamente superior en los participantes del C.S. rural (mediana de 8 años) respecto al urbano y semiurbano $(p<0,0001)$, (figura 1).

En cuanto al índice de masa corporal, únicamente se observaron diferencias entre el centro de salud rural y urbano, con una media de 29,8 en el rural frente a 32,8 en el urbano $(p=0,03)$ (figura 2$)$.

Como podemos observar en la tabla 2, que hace referencia a la proporción de pacientes que tenían las exploraciones protocolizadas, en el centro de salud rural figuraba el registro de la realización de fondo de ojo en el $46,7 \%$ de los pacientes, electrocardiograma en el $73,3 \%$, determinación de microalbuminuria en el $37,8 \%$, y de hemoglobina glicosilada cada 6 meses en el $86,7 \%$, siendo estas proporciones significativamente superiores a las de los otros centros. Un $82 \%$ de los pacientes del centro rural y un $78,6 \%$ de los pacientes del semiurbano mostraron una hemoglobina glicosilada $<7 \%$ (tabla 2).

Finalmente, por medio de un análisis de regresión logística se identificó como única variable independiente asociada al seguimiento del protocolo el pertenecer al centro de salud rural: urbano en relación al rural OR: 0,18 (IC95\%: 0,06-0,56) y semiurbano en relación al rural OR: 0,18 (IC95\%: 0,05-0,58).

\section{DISCUSIÓN}

Nuestros resultados muestran una mediana de hemoglobina glicosilada de $7 \%$ en el centro de salud urbano, que es superior al valor de la mediana de este parámetros en los centros de salud semiurbano y rural. Esto traduce un control metabólico de la diabetes tipo 2 más deficiente en el centro de salud urbano, evaluado mediante las cifras de $\mathrm{HbA} 1 \mathrm{c}$.

Como parámetros para evaluar la calidad de la asistencia sanitaria al igual que en la mayor parte de estudios revisados hemos utilizado como indicadores de proceso la determinación de $\mathrm{HbA1c}$ anual, perfil lipídico, microalbuminuria, realización de fondo de ojo ${ }^{9,12-14}$, sin considerar la exploración 


\begin{tabular}{l|cccc}
\hline VARIABLES & $\begin{array}{c}\text { C. S. } \\
\text { URBANO }\end{array}$ & $\begin{array}{c}\text { C.S. } \\
\text { SEMIURBANO }\end{array}$ & $\begin{array}{c}\text { C. S. } \\
\text { RURAL }\end{array}$ & TOTAL \\
\hline $\begin{array}{l}\text { Sexo (n, \%) } \\
\text { Mujeres } \\
\text { Hombres }\end{array}$ & $32(38,6 \%)$ & $26(57,8 \%)$ & $20(44,4 \%)$ & $78(45,1 \%)$ \\
& $51(61,4 \%)$ & $19(42,2 \%)$ & $25(55,5 \%)$ & $95(54,9 \%)$ \\
Edad al diagnóstico & & & & \\
[Media, ( DE)] & $68,9(12,1)$ & $66,1(11,5)$ & $70,4(11,7)$ & $68,6(11,9)$ \\
\hline
\end{tabular}

Tabla 1. Distribución de frecuencias de las variables sociodemográficas consideradas en el estudio.

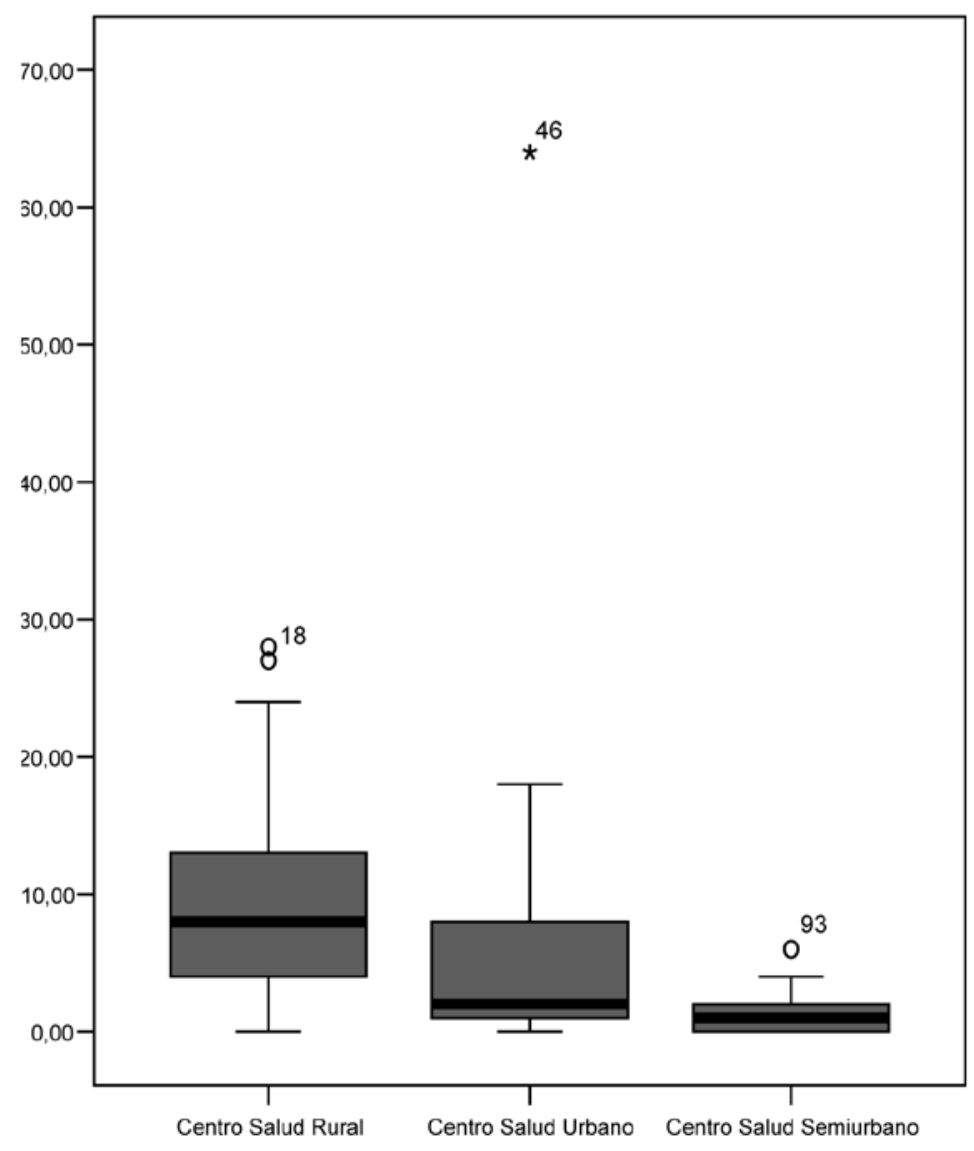

Figura 1. Distribución de los valores del tiempo de evolución para los Centros de Salud Rural, Urbano y Semiurbano. 


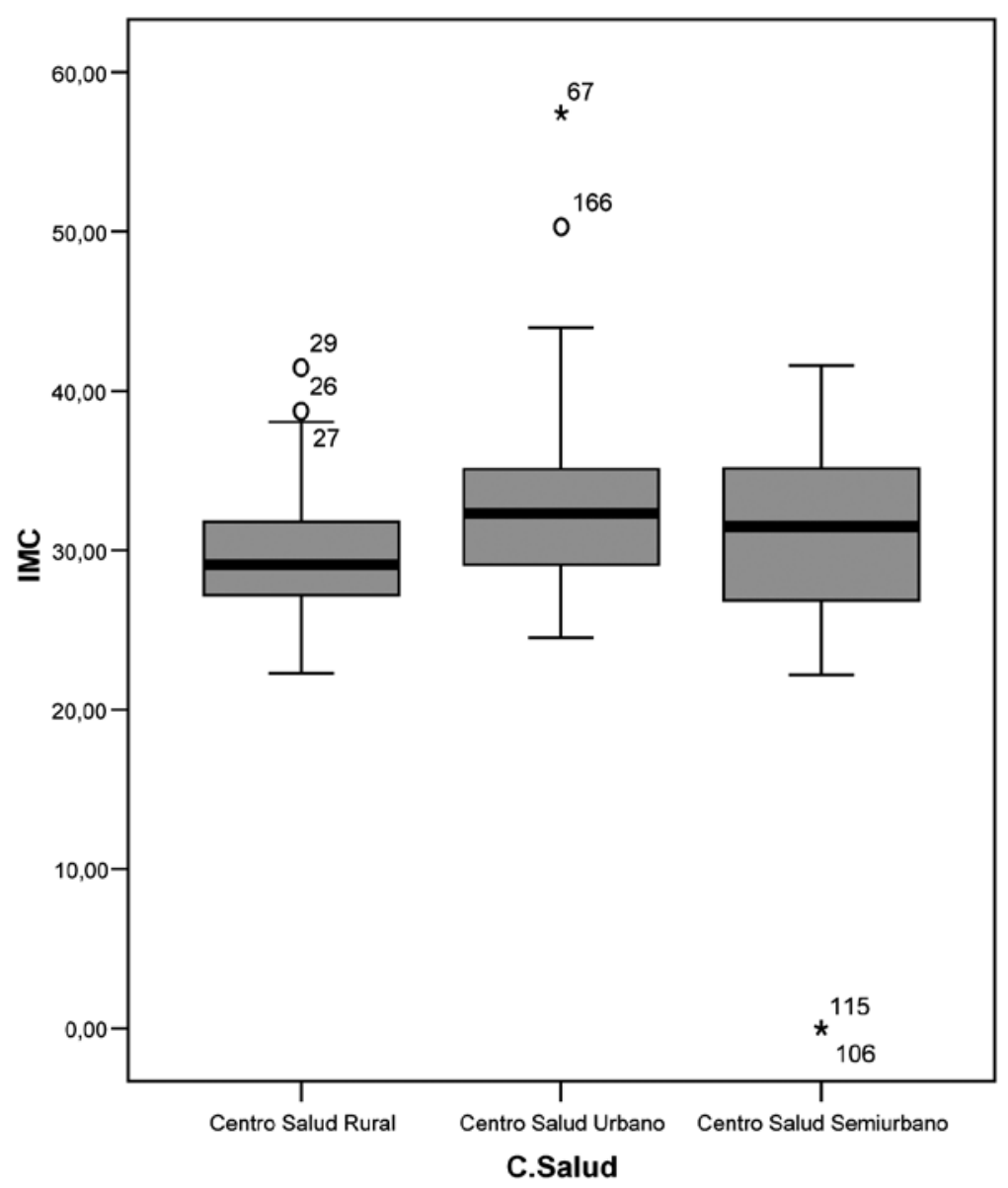

Figura 2. Distribución de los valores del Indice de Masa Corporal (IMC)

\begin{tabular}{l|ccccc}
\hline & $\begin{array}{c}\text { C.S. } \\
\text { URBANO }\end{array}$ & $\begin{array}{c}\text { C.S. } \\
\text { SEMIURBANO }\end{array}$ & $\begin{array}{c}\text { C. S. } \\
\text { RURAL }\end{array}$ & TOTAL & P \\
\hline Fondo de ojo & $18,1 \%$ & $15,6 \%$ & $46,7 \%$ & $24,9 \%$ & 0,0001 \\
Electrocardiograma & $18,1 \%$ & $42,2 \%$ & $73,3 \%$ & $18,1 \%$ & $<0,0001$ \\
Microalbuminuria & $34,9 \%$ & $11,1 \%$ & $37,8 \%$ & $29,5 \%$ & 0,007 \\
Hemoglobina glicosilada & $53,0 \%$ & $46,7 \%$ & $86,7 \%$ & $53 \%$ & $<0,0001$ \\
Hemoglobina glicosilada en rango & $53,7 \%$ & $78,6 \%$ & $82 \%$ & $69,6 \%$ & $<0,0001$ \\
Hemoglobina glicosilada cada 6 & $(\mathrm{~N}=54)$ & $(\mathrm{N}=42)$ & $(\mathrm{N}=39)$ & $(\mathrm{N}=135)^{*}$ & $<$ \\
meses & $53,0 \%$ & $46,7 \%$ & $86,7 \%$ & $60,1 \%$ & $<0,0001$ \\
\hline
\end{tabular}

Tabla 2. Proporción de pacientes que tienen realizadas las exploraciones protocolizadas. * En 38 pacientes no dispusimos de la HbA1c y se excluyeron del análisis 
del pie diabético, pero incluyendo la realización o no de electrocardiograma, como lo valoraron previamente López et al ${ }^{13}$.

No hemos encontrado en la literatura revisada ningún estudio en el que se establezca la comparación en cuanto al seguimiento de pacientes con diabetes mellitus tipo 2 en diferentes contextos de atención primaria. En nuestro estudio, en tres centros de salud, urbano, semiurbano y rural, hemos constatado diferencias, en cuanto a la proporción de pacientes que tienen realizadas las exploraciones protocolizadas, a favor del centro de salud rural.

La media de edad de nuestros pacientes era similar a la descrita en el resto de estudios analizados. El perfil del paciente con diabetes tipo 2 atendido en atención primaria es el de un sujeto de alrededor de 68 años y que es obeso (IMC alrededor de 30 ), con un promedio de hemoglobina glicosilada aceptable ${ }^{9,13}$.

En contraste con nuestros hallazgos de un mejor control metabólico en el centro de salud rural, la literatura muestra resultados dispares. Díaz et a ${ }^{15}$ valoraron el cumplimiento de los objetivos de control metabólico en pacientes con diabetes tipo 2 en atención primaria de ámbito rural, comparándolo con los valores recomendados por la American Diabetes Association (ADA) y el Grupo de estudio de la diabetes en atención primaria de salud (GEDAPS). Los resultados obtenidos para la hemoglobina glicosilada fueron aceptables (en aquellos a los que se les había determinado), situándose en el límite del objetivo de buen control. Sin embargo, casi un $20 \%$ de la muestra no disponía del registro de una determinación de hemoglobina glicosilada en los últimos 6 meses, de forma similar a lo mostrado tanto en nuestros resultados como en los de Coon et $\mathrm{al}^{18}$.

En este estudio hemos observado diferencias en la realización de hemoglobina glicosilada entre el ámbito rural, urbano y semiurbano, mostrando resultados similares respecto al control de la glucemia medida con la hemoglobina glicosilada como se observa en los estudios de Benito et $\mathrm{al}^{19}$ y Virgós et $\mathrm{al}^{20}$. Para Carral et $\mathrm{al}^{12} \mathrm{y}$ López et $\mathrm{al}^{13}$ el grado de cumplimiento en la determinación anual de microalbuminuria, perfil lipídico completo y exploración de pies también fue insuficiente, sin embargo, el porcentaje de determinación anual de hemoglobina glicosilada fue aceptable, refiriendo que sólo al $31,5 \%$ de los pacientes se les realizó fondo de ojo y sólo a la mitad se les realizó electrocardiograma. Estos porcentajes son similares a los de nuestro estudio en el que indica que se realizó fondo de ojo al $25 \%$ de los pacientes y un electrocardiograma al $39 \%$ de ellos. En la investigación publicada por Gallego ${ }^{8}$, se encontraron déficits en relación con la determinación de hemoglobina glicosilada, albuminuria y realización de fondo de ojo. Rubio et $\mathrm{a}^{21}$ observaron que una buena parte de los pacientes diabéticos seguidos presentan indicadores de proceso situados por debajo de lo que se recomienda en los consensos, aunque existen algunos indicadores no valorados como la exploración de pies y la realización de fondo de ojo.

En otras investigaciones como la de Arroyo et $\mathrm{al}^{2}$, se evidencia que en una proporción importante de los pacientes con diabetes tipo 2 , incluso en aquellos con más tiempo de evolución, no se explora la presencia de nefropatía, de retinopatía ni de neuropatía periférica. El alto porcentaje de pacientes no explorados alerta sobre la necesidad de concienciar en la prevención de las complicaciones crónicas. Observaron que los pacientes recién diagnosticados de diabetes mellitus tipo 2 fueron a quienes se practicaron más frecuentemente la determinación de hemoglobinas glicosiladas, perfil lipídico, microalbuminurias y la realización de fondos de ojo. Esto coincide con los resultados publicados por Brown et $a^{22}$ que señala que los incrementos en los costes en Salud en los pacientes con diabetes tipo 2 son evidentes tras el diagnóstico, y ya en el primer año son 2,1 veces superiores que los costes en salud de los pacientes sin diabetes.

Por otra parte, López et $\mathrm{al}^{13}$ demostraron que los pacientes atendidos en Endocrinología presentaban significativamente mejor cumplimentación en la determinación de hemoglobina glicosilada y en la realización de fondo de ojo, pero también observaron mayor proporción de pacientes atendidos en Atención Primaria con niveles de hemoglobina glicosilada $<6,5 \%$. Sin embargo, Carral et $\mathrm{al}^{12}$ encontró que los pacientes en seguimiento por Atención Primaria eran de mayor edad que los seguidos por Atención Especializada.

Un reciente estudio ${ }^{14}$ presentó los resultados de las evaluaciones sucesivas de la calidad asistencial en diabetes tipo 2 en España, según las recomendaciones del programa de mejora continua de la calidad (MCC) de la RedGedaps, de los años 1996, 1998, 2000, 2002 y 2007. Los autores concluyeron que se ha registrado un aumento en todas las determinaciones analíticas (hemoglobina glicosilada, 
perfil lipídico, microalbuminuria) desde el año 1996 hasta el año 2007. Las exploraciones físicas anuales (fondo de ojo, exploración de pies) se efectúan en aproximadamente la mitad de los diabéticos, comprobando un incremento progresivo de las exploraciones oftalmológicas hasta el 2007, pero sin apreciar cambios significativos en las exploraciones de pies. Existen problemas que se detectaron repetidamente y que ocasionaron un consenso sobre la priorización de las siguientes medidas correctoras: aumentar el uso de la hemoglobina glicosilada como marcador de control glucémico, hasta alcanzar un mínimo de dos determinaciones anuales y sistematizar el hábito de la exploración de pies del paciente diabético en las visitas de control (al menos cada 12 meses). A los 10 años del programa se han observado reducciones de retinopatía diabética, de úlceras de pie diabético y amputaciones y de las complicaciones macrovasculares.

Una posible limitación de nuestro estudio podría ser la ausencia de datos en la historia clínica que podría subestimar las actuaciones realizadas sobre el paciente.

En conclusión, nuestros resultados muestran que existe un mejor seguimiento de los pacientes diabéticos en el medio rural que en el urbano y semiurbano. Esto podría estar en relación con la mayor demanda asistencial que existe en el Centro de Salud Urbano, lo que dificulta el control estricto de las patologías crónicas como la diabetes. Además, los pacientes son seguidos en muchas ocasiones por otros especialistas a los que tienen más fácil acceso que desde el medio rural, lo que puede ocasionar que no acudan tanto a los controles por Atención Primaria.

\section{BIBLIOGRAFIA}

1. World Health Organization. Definition, Diagnosis and classification of diabetes mellitus and its complications: Report of a WHO Consultation. Part1: Diagnosis and clasification of diabetes mellitus. Geneve: World Heath Organization; 1999.

2. Arroyo J, Badía X, de la Calle H, Díez J, Estmatjes E, Fernández $\mathrm{I}$, et al. Tratamiento de los pacientes con diabetes mellitus tipo 2 en atención primaria en España. Med Clin (Barc). 2005; 125:166-72.

3. Ministerio de Sanidad y Consumo. Estrategia en diabetes del Sistema Nacional de Salud. (Consultado el 25 de febrero de 2010). Disponible en www.msc.es
4. Calderón, A. Epidemiología, genética y mecanismos patogénicos de la diabetes mellitus. Rev Esp Cardiol. 2007;7:3H-11H.

5. Valdés S, Rojo G, Soriguer F. Evolución de la prevalencia de la diabetes tipo 2 en población adulta española. Med Clin (Barc). 2007;129:352-5.

6. Martín A. Atención primaria y diabetes mellitus tipo 2: limitaciones actuales del cribado y las acciones comunitarias. Endocrinol Nutr. 2009;56:51-2.

7. Martínez A, Leal M, Martínez A, Navarro AF, Lifante Z, Gómez $\mathrm{M}$. Efectos de un programa de revisión en el control de los diabéticos tipo 2 seguidos en atención primaria. Programa Diabetes First. Endocrinol Nutr. 2010;57:16-21.

8. Gallego, RJ. Calidad de la asistencia al paciente con diabetes mellitus tipo II en el ámbito de una consulta de Atención Primaria. Av Diabetol. 2002;18:84-93.

9. Goday A, Delgado E, Díaz F, De Pablos P, Vázquez JA, Soto E. Epidemiología de la diabetes tipo 2 en España. Endocrinol Nutr. 2002;49:113-26.

10. Ortiz MT, Orozco D, Gil V, Terol C. Frecuentación y grado de control del paciente diabético tipo 2. Aten Primaria. 2008;40:139-44.

11. Gancedo J, Carrera E, Alconchel S, Delso Al. Seguimiento de pacientes con diabetes mellitus tipo 2 en un centro de Salud. Semergen. 2006;32:265-72.

12. Carral F, Ballesta MJ, Aguilar M, Ortego J, Torres I, Garcia $A$, et al. Evaluación de la calidad asistencial en pacientes con diabetes tipo 2 del Área Sanitaria Cádiz-San Fernando. Av Diabetol. 2005;21:52-7.

13. López I, Luna MR. Evaluación de calidad de control asistencial de diabéticos tipo 2 en un área de salud Pontevedra-Sur. Cad Aten Primaria. 2009;16:8-11.

14. Franch J. Persiguiendo la mejora continua en la calidad de la asistencia a las personas con diabetes tipo 2. La experiencia de la RedGedaps. Revista Diabetes Práctica. 2010;1(Supl. 1):33-43.

15. Díaz GJ, Palmeiro G, Casado I, Arandia M, Portuburu MM; Vázquez LA. Cumplimiento de los objetivos de control metabólico en diabetes mellitus en el medio rural de Ourense. Rev Esp Salud Pública. 2006;80:67-75.

16. Grupo de Estudio de la Diabetes en Atención Primaria de Salud (GEDAPS) de la Sociedad Catalana de Medicina Familiar y Comunitaria. Protocolo de Diabetes tipo 2. (Consultado el 26 de abril de 2009). Disponible en www. sediabetes.org.

17. American Diabetes Association. Standars of Medical Care in diabetes. Diabetes Care. 2010;33:S4-S10.

18. Coon P, Zulkowski K. Adherence to American Diabetes Association Standars of Care by Rural Health Care Providers. Diabetes Care. 2002; 25:2224- 29.

19. Benito P, García R, Puig M, Mesa J, Pallardo LF, Faure E. Perfil de los pacientes con diabetes mellitus tipo 2 en la Atención Primaria española. Rev Clin Esp. 2004;204:1-2.

20. Virgós M, Castro M, Martínez S, Vila A. ¿Hacemos caso del resultado de la hemoglobina glicosilada a la hora tratar a nuestros pacientes diabéticos?. Aten Primaria. 2002;30:157-62.

21. Rubio JA, Arribas I, Peláez N, Maqueda E, Peña V, García A. Resultados analíticos en la población con diabetes del área 3 de Madrid: un análisis de calidad asistencial atendiendo al GRD. Av Diabetol. 2005;21:323-30.

22. Brown JB, Nichols GA, Glauber HS, Bakst AW. Type 2 diabetes: incremental medical care cost during the first 8 years after diagnosis. Diabetes Care. 1999;22:1116-24. 